\title{
Landscape connectivity analysis for conservation: insights from combining new methods with ecological and genetic data
}

\author{
Sandra Luque $\cdot$ Santiago Saura $\cdot$ \\ Marie-Josée Fortin
}

Received: 8 December 2011 / Accepted: 14 December 2011/Published online: 3 January 2012

(C) Springer Science+Business Media B.V. 2011

Landscape connectivity is a multi-scalar concept allowing the investigation of how the interaction between species movement abilities and landscape structure affects species survival, gene flow and other key ecological processes in fragmented landscapes. This requires the determination of functional connectivity which is the end result of a complex combination of multiple factors such as habitat amount and arrangement, matrix quality and permeability, species perceptions and dispersal behaviour, population density, etc. Functional connectivity quantification necessitates also the consideration of the impacts and constraints imposed by the increasing rates of landscape and environmental change, which are ultimately driven by socioeconomic factors and are likely to continue putting more pressures on both managed and natural landscapes.

\section{S. Luque $(\bowtie)$}

Cemagref, Institute for Agricultural and Environmental Engineering Research, Mountain Ecosystems Research Unit, 2 Rue de la Papeterie, 38402 Saint-Martin d'Hères, France

e-mail: sandra.luque@irstea.fr; sandra.luque@cemagref.fr

\section{S. Saura}

ETSI Montes, Universidad Politécnica de Madrid, Madrid, Spain

\section{M.-J. Fortin}

Department of Ecology and Evolutionary Biology, University of Toronto, Toronto, Canada
Connectivity is nowadays an important concern in almost any modern conservation plan around the globe. The challenge of these conservation plans is to identify the spatial scale(s) and key landscape elements needed to maintain or restore connectivity and the ecological processes that are promoted by it. To meet the final objective of favouring species viability and ecosystem diversity, landscape ecologists should be able to deliver conservation guidelines and indicators at the spatial scale at which the impacts of landscape change are most prominently affecting the abundance and persistence of the focal species. They also need to convincingly demonstrate the effectiveness and benefits of connectivity investments compared to other competing conservation alternatives. In this context, integrated approaches offering synergies and new capabilities for connectivity conservation planning should allow us to interpret the effects of landscape spatial heterogeneity and to define critical threshold levels at which landscapes are connected allowing the persistence of species (Opdam et al. 2003).

The series of papers in this special issue constitute a valuable addition towards this end, by showing how a diversity of analytical approaches and data types can be applied, and in many case combined in an integrated fashion, in order to address various aspects that are at play while quantifying landscape connectivity and to support related management decisions. The contributions from this special issue include (i) empirical assessments of the role of different connectivity-related 
factors on species occurrence and distribution (Awade et al.; Lizée et al.; Rico et al.; Zozaya et al.); (ii) analyses of the impacts on species genetic diversity of the interactions between climate change and limited landscape connectivity (Cobben et al.; Wasserman et al.), (iii) insights on how landscape heterogeneity and permeability for pollinators determine the genetic structure of tree populations (Dyer et al.) and on the factors influencing the robustness of the correlation between ecological and genetic distances (Graves et al.) (iv) evaluations of the contribution of different linkages and habitat areas to sustain landscape connectivity and promote species survival and potential range expansion (Carranza et al.; Decout et al.); (v) the development of methods for cost-efficient linkage design based on environmental variables as surrogates for multiple species-specific connectivity needs and responses (Alagador et al.); and (vi) a review and conceptual framework providing insights on the potential and possibilities of a spatial graph approach to support the conservation of freshwater habitats (Erös et al.).

\section{Graph theory and habitat availability metrics as conceptual frameworks to study functional connectivity}

Graph-theoretical approaches have been promoted in the last decades (Dale and Fortin 2001; Urban and Keitt 2001; Urban et al. 2009) as analytical tools to study the effects of landscape fragmentation on animal movement and species persistence as well as to optimize the selection of reserve networks. In particular, graph structures have been shown to be a powerful and effective way of both modelling landscape networks and performing complex analysis regarding connectivity and population persistence across the landscape (Fall et al. 2007; Minor and Urban 2007; Saura and Pascual-Hortal 2007). They provide a spatially explicit representation of the landscape that is able to evaluate the contribution to connectivity of individual landscape elements. In most cases graph theory makes very similar predictions to more complex and data-hungry spatially explicit population models, and may provide some additional insights not available from the latter (Minor and Urban 2007; Visconti and Elkin 2009). To advance in our understanding of species functional responses to landscape heterogeneity, the dynamic property of landscape connectivity at different scales needs to be integrated through multi-scalar properties of the entire network not simply aggregating properties of a set of individual patches. Different graph-based methods and metrics are applied, reviewed or developed in seven papers in this special issue (Alagador et al.; Awade et al.; Erös et al.; Carranza et al.; Decout et al.; Dyer et al.; Zozaya et al.), which offer a good testimony of the increasing breadth and acceptance of this analytical approach by the landscape ecology community.

Recent improved graph-based metrics for the integration of connectivity in conservation planning were developed based on the concept of measuring habitat availability (reachability) at the landscape scale (Pascual-Hortal and Saura 2006; Saura and Pascual-Hortal 2007; Saura and Rubio 2010). This concept of habitat availability consists in considering a patch itself as a space where connectivity occurs; connectivity is then considered as a property of the landscape that allows species to reach (to have available) a larger amount of the habitat resources existing in the landscape, no matter if such reachable habitat comes from a big or high quality patch itself (intrapatch connectivity), from the strong connections between different patches (interpatch connectivity) or, more frequently, from a combination of both. This habitat availability approach helps to place connectivity considerations in a broader and more informative context of conservation management alternatives and to avoid arbitrary decisions on the weight that connectivity per se should have in the final planning (Saura and Rubio 2010). Four of the graph-based papers in this special issue apply either the IIC (Integral Index of Connectivity) or the PC (Probability of Connectivity) metrics, their fractions, or their related ECA (Equivalent Connected Area) metric (Saura et al. 2011).

The set of papers in this issue include probabilistic connectivity analysis in which no sharp distance threshold is used to determine the links in the graph (Awade et al.; Carranza et al.; Zozaya et al.), applications of spatial graphs to large-scale grid-based data on the distribution of habitat and environmental variables (Alagador et al.; Zozaya et al.), and applications of methods that evaluate the different roles of habitat patches in the landscape network allowing to determine the final conservation priorities while avoiding an overemphasis in the relevance of 
interpatch connectivity (Carranza et al.). This might alleviate some of the concerns timely raised by Moilanen (2011), together with other previous studies that have shown how some graph-based approaches provide improved and solid ways to account for the contribution of multiple paths in connectivity analysis (McRae et al. 2008; Pinto and Keitt 2009). Indeed, spatial graphs in ecology have already shaped a very broad field with a diversity of methods, metrics and approaches (Erös et al.), which cover a wide range of possibilities and analytical tools which should be carefully selected for adequately meeting the needs of different research question and conservation goals.

\section{Functional connectivity in terrestrial and aquatic ecosystems}

Despite mounting evidence that both habitat spatial configuration and the intervening matrix influence species persistence in fragmented landscapes, the relative importance of each of these factors in determining spatial population structure remains poorly understood. Moreover, wildlife conservation in human-dominated landscapes is challenging, frequently involving lengthy and costly negotiations with different stakeholders to preserve the integrity of terrestrial systems. Wasserman et al. provided an evaluation on the effects of warming climate on landscape resistance, gene exchange and population connectivity for the American marten in the northern Rocky Mountains (USA). Awade et al. showed how probabilistic habitat availability indices (parameterized through playback techniques) are effective in explaining bird species occurrence patterns in fragmented forests ecosystems in Brazil, while Lizée et al. analyzed how matrix configuration and patch isolation influence species-area relationship for urban butterfly communities in France. Zozaya et al. showed how the shifting habitat patterns and connectivity relationships regulated by forest fires are key factors to understand bird species distribution and their dynamics in fireprone Mediterranean areas. Alagador et al. identified linkages between environmentally-similar protected areas in the Iberian Peninsula (Natura 2000 network), providing an original method for optimising connectivity based on a variant of the minimum Steiner tree problem in graphs.
Several stimulating examples related to the connectivity and functioning of riverine and freshwater habitats can be found as well in the present issue. Carranza et al. analysed the capacity of species to move both on freshwater and terrestrial realms in the case of the endangered Eurasian otter in Italy. Advances in the application of graph theory and habitat availability metrics were also used to explore and provide a connectivity analysis framework that integrates habitat suitability and dispersal responses to landscape permeability (Decout et al.). This contribution, with the common frog as the focal species, determined a key role for landscape-based approaches in identifying "availability-suitability-connectivity" patterns from local to regional, providing an operational tool for landscape planning and conservation. Erös et al. highlighted the potential application of graph theory in freshwater ecology and conservation. This contribution provides an overview of the potential of hierarchical patch-based spatial graphs combined with a conceptual framework for topological analysis of stream networks (i.e. riverscape graphs).

\section{Using genetic data to test the effects of landscape connectivity}

Landscape genetics provides an ideal framework to address the question of functional connectivity, by investigating the effect of landscape on process and patterns of gene flow. A large amount of recent work analyses the effect of landscapes on genetic structure, by correlating genetic distances to landscapes distances reflecting resistance to movement (e.g. Cushman et al. 2006; Stevens et al. 2006; Landguth et al. 2010). However, a first milestone to provide realistic measures of dispersal to be integrated in graph and structural landscape analysis is to remove history from usual measures of gene flow, in order to provide estimates of current gene flow (e.g. Dyer and Nason 2004; Sork et al. 2005). Genetic connectivity by itself cannot fully describe functional connectivity (e.g. Lowe and Allendorf 2010), demographic information is needed (population size, dispersal, etc.) as well. At present, studies integrating both demographic and genetic measures of connectivity are scarce (but see Gaggiotti et al. 2002, 2004; Foll and Gaggiotti 2006; Stevens et al. 2006; Faubet and Gaggiotti 2008; 
Gauffre et al. 2008 for approaches that allow such integration). We are not trying here to fulfil this gap, but to provide a better understanding of the effects of scale on measuring functional connectivity while at the same time provide better integration of functional and structural connectivity measures. For instance, in fragmented landscapes, plant species persistence depends on functional connectivity in terms of pollen flow to maintain genetic diversity within populations, and seed dispersal to re-colonize habitat patches following local extinction. Rico et al. provided an example for calcareous grassland communities, claiming the importance to test alternative dispersal vectors. While Dyer et al. propose an example on genetic connectivity in the North American understory tree, Cornus florida, quantifying pollen pool covariance networks and the influence of the intervening landscape. Graves et al. provided insights from a simulation study on the impact of landscape characteristics and spatial errors on the strength and variability of landscape genetics inference.

\section{Future directions}

Maintaining landscape connectivity is an essential aspect of landscape management for biodiversity. We need to consider and implement connectivity recommendations carefully in each landscape plan by adequately selecting the most appropriate approaches, methods and input data for the analysis. Hence experience, further advances in research, and monitoring of our efforts will allow us to improve our understanding and refine how to manage our natural and human-modified systems to maintain landscape connectivity at the scale of the interaction between the organisms and the landscape.

The papers in this special issue on landscape connectivity represent a compelled significant interdisciplinary collaboration where the authors provide a wide range of examples of connectivity and functioning of complex system in different regions and different systems of the world. Still key research questions remain open for future efforts, in particular on:

(1) Study the spatial dynamics of landscape connectivity using spatio-temporal graphs and network dynamics analysis;
(2) Improve methods and analytical tools to identify key habitat areas and links that most efficiently uphold connectivity at different spatial and temporal scales. This should contribute to implement appropriate conservation guidelines that include the effect of landscape dynamics, environmental change and species dispersal abilities.

(3) Further contribute to place connectivity together with other alternative conservation possibilities in a broader analytical framework to guide ecologists and conservationists. This should avoid overemphasizing (or underestimating) the importance of connectivity in order to efficiently meet management and conservation goals.

(4) Acquire more and better quality empirical data on species movement, habitat use, genetic patterns, etc. for adequately feeding the graph models and the connectivity analyses in general.

(5) Couple spatial networks with species networks (metanetworks) to better understand how the impacts of landscape structure in ecosystem functioning are modulated by species interactions such as predation, pollination or competition.

(6) Develop tools for making the theoretical and methodological developments on landscape connectivity available for (and usable by) practitioners.

\section{References}

Cushman SA, McKelvey KS, Hayden J, Schwartz MK (2006) Gene flow in complex landscapes: testing multiple hypotheses with causal modeling. Am Nat 168:486-499

Dale MRT, Fortin M-J (2001) From graphs to spatial graphs. Annu Rev Ecol Evol Syst 41:21-38

Dyer RJ, Nason JD (2004) Population graphs: the graph-theoretic shape of genetic structure. Mol Ecol 13:1713-1728

Fall A, Fortin M-J, Manseau M, O’Brien D (2007) Spatial graphs: principles and applications for habitat connectivity. Ecosystems 10:448-461

Faubet P, Gaggiotti OE (2008) A new Bayesian method to identify the environmental factors that influence recent migration. Genetics 178:1491-1504

Foll M, Gaggiotti OE (2006) Identifying the environmental factors that determine the genetic structure of populations. Genetics 174:875-891

Gaggiotti OE, Jones F, Amos W, Harwood J, Nichols RA (2002) Patterns of colonisation in a grey seal metapopulation. Nature 416:424-427

Gaggiotti OE, Brooks SP, Amos W, Harwood J (2004) Combining demographic, environmental and genetic data to test hypotheses about colonization events in metapopulations. Mol Ecol 13:811-825 
Gauffre B, Estoup A, Bretagnolle V, Cosson JF (2008) Spatial genetic structure of a small rodent in a heterogeneous landscape. Mol Ecol 17:4619-4629

Landguth EL, Cushman SA, Murphy MA, Luikart G (2010) Relationships between migration rates and landscape resistance assessed using individual-based simulations. Mol Ecol Resour 10:854-862

Lowe WH, Allendorf FW (2010) What can genetics tell us about population connectivity? Mol Ecol 19:3038-3051

McRae BH, Dickson BG, Keitt TH, Shah VB (2008) Using circuit theory to model connectivity in ecology, evolution, and conservation. Ecology 89:2712-2724

Minor ES, Urban DL (2007) Graph theory as a proxy for spatially explicit population models in conservation planning. Ecol Appl 17:1771-1782

Moilanen A (2011) On the limitations of graph-theoretic connectivity in spatial ecology and conservation. J Appl Ecol 48:1543-1547

Opdam P, Verboom J, Pouwels R (2003) Landscape cohesion: an index for the conservation potential of landscapes for biodiversity. Landscape Ecol 18:113-126

Pascual-Hortal L, Saura S (2006) Comparison and development of new graph-based landscape connectivity indices: towards the priorization of habitat patches and corridors for conservation. Landscape Ecol 21:959-967

Pinto N, Keitt T (2009) Beyond the least-cost path: evaluating corridor redundancy using a graph-theoretic approach. Landscape Ecol 24:253-266
Saura S, Pascual-Hortal L (2007) A new habitat availability index to integrate connectivity in landscape conservation planning: comparison with existing indices and application to a case study. Landsc Urban Plan 8:91-103

Saura S, Rubio L (2010) A common currency for the different ways in which patches and links can contribute to habitat availability and connectivity in the landscape. Ecography 33:523-537

Saura S, Estreguil C, Mouton C, Rodríguez-Freire M (2011) Network analysis to assess landscape connectivity trends: application to European forests (1990-2000). Ecol Indic 11:407-416

Sork VL, Dyer RG, Smouse PE, Apsit VJ, Westfall RD (2005) A two-generation analysis of pollen structure in flowering dogwood (Cornus florida L.) in the Missouri Ozarks. Am J Bot 92:262-271

Stevens VM, Verkenne C, Vandewoestijne S, Wesselingh RA, Baguette M (2006) Gene flow and functional connectivity in the natterjack toad. Mol Ecol 15:2333-2344

Urban DL, Keitt TH (2001) Landscape connectivity: a graphtheoretic perspective. Ecology 82:1205-1218

Urban DL, Minor ES, Treml EA, Schick RS (2009) Graph models of habitat mosaics. Ecol Lett 12:260-273

Visconti P, Elkin C (2009) Using connectivity metrics in conservation planning - when does habitat quality matter? Divers Distrib 15:602-612 